\title{
IL CONTRIBUTO DEI SERVIZI IDRICI INTEGRATI AL FABBISOGNO IRRIGUO DEI COMPRENSORI AGRICOLI: IL CASO DI MILANO
}

\author{
MAURIZIO BROWN (*)
}

SunTO. - Nel corso della sua storia Milano ha saputo far tesoro delle proprie acque di rifiuto raccolte dalla roggia Vettabbia. Dal XII secolo, grazie anche all'opera dei monaci delle Abbazie di Chiaravalle e Viboldone, si sarebbe diffuso l'uso di spandere la acque luride della Vettabbia sui prati marciatori di un ampio comprensorio agricolo a valle della città, recuperando i nutrienti in esse contenuti e massimizzando la produzione di foraggio fresco. Nella seconda metà del XIX secolo questa pratica fu perfezionata e resa più efficiente per consentire lo smaltimento sostenibile delle acque cloacali raccolte dal nuovo sistema fognario della città. Dal 2004 Milano si è dotata di un efficace sistema di depurazione che consente il riutilizzo delle acque depurate in agricoltura, diventando in questo campo l'esempio più significativo in Europa.

$* * *$

ABSTRACT. - During its history Milan city has been able to treasure its wastewater collected by Vettabbia irrigation ditch. Since the XII century, also thanks to Cistercensi (monks from Chiaravalle Abbey) great endeavour, the use of effusing the filthy waters from Vettabbia over the rotten lawns of a great and huge agricultural district, situated in the South the City, was going to become the most spread method used to regain the nutrients contained into the wastewaters and, at the same time, a great procedure to optimize the fresh forage production. During the second half of XIX century this technique was refined and made more efficient in order to allow the sustainable and ecofriendly disposal of the drainage waters raised from the new sewerage system of the City. Since 2004 Milan adopted an effective water treatment plant which permits the reuse of the purified waters for what concerns agriculture, becoming, in this field, the most significant model in Europe.

${ }^{(*)}$ Già Direttore Acque Reflue di Metropolitana Milanese. Servizio Idrico Integrato della Città di Milano, Italia. E-mail: maurizio.brown@libero.it 


\section{FARE TESORO DELle ACQue REFLUe}

Le acque reflue urbane, ovvero le acque usate o di rifiuto, provenienti dalle attività umane in generale e quelle di origine meteorica, sono state generalmente considerate un rifiuto fastidioso e ingombrante da smaltire rapidamente lontano dagli insediamenti civili.

In alcune realtà le acque usate sono state invece valorizzate e trasformate in una risorsa divenendo un fattore di sviluppo: è il caso di Milano.

La nostra città sorge al centro di una vasta regione pianeggiante, compresa tra le Prealpi e il Po, delimitata a oriente e a occidente, rispettivamente dai fiumi Adda e Ticino, ricca di acque superficiali e sotterranee, ma lontana dai fiumi principali.

I suoi abitanti compresero che lo sviluppo del loro insediamento e il loro benessere erano in buona parte condizionati dall'abbondanza di acqua, particolarmente necessaria agli usi civili, al recupero di nuove aree agricole, al perfezionamento e alla differenziazione delle coltivazioni, alla difesa della città (fossati), oltre che per ricavarne forza motrice (mulini) e per il trasporto delle merci.

S'impegnarono innanzitutto a realizzare un'ampia opera di bonifica del territorio, imbrigliando le acque risorgive e i piccoli corsi d'acqua esistenti, inalveandole e destinandole principalmente all'uso irriguo (fontanili, rogge, marcite).

Più tardi, probabilmente già in epoca romana, furono attuati interventi più radicali, mirati a deviare verso la città i due corsi d'acqua più consistenti presenti nel vasto e ricco bacino circostante.

Risulta ormai certo che i corsi del fiume Olona e del torrente Seveso furono deviati per alimentare con le loro acque il fossato di difesa della città romana (Fig. 1).

Il principale emissario delle acque cittadine divenne la roggia Vettabbia, un corso d'acqua di origine antichissima che ha l'incile nel centro storico di Milano, nei pressi di via Molino delle Armi, sviluppandosi poi in direzione sud orientale, toccando in successione le località di Nosedo, Chiaravalle e Viboldone, per immettersi, infine, nel fiume Lambro Settentrionale poco a monte di Melegnano.

La Vettabbia raccolse prima le acque della fossa difensiva dell'epoca romana (Canali Piccolo e Grande Sevese) e in seguito anche quelle del fossato medioevale (Cerchia dei Navigli o Fossa Interna).

Ben presto la roggia finì per smaltire anche gran parte delle acque di rifiuto della città che venivano scaricate nei due fossati citati. 


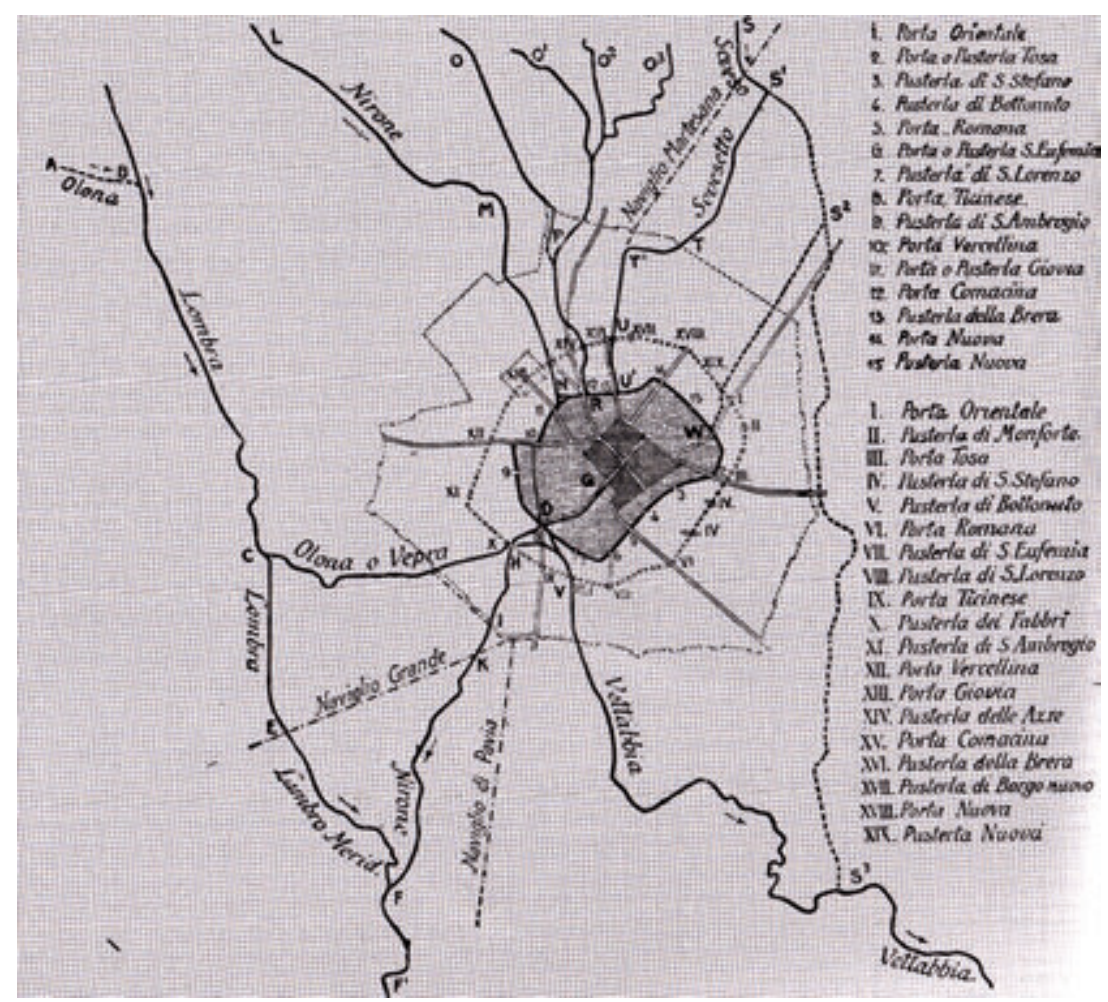

Fig. 1. Ricostruzione dell' idrografia antica del territorio milanese tratta da Felice Poggi.

La fognatura di Milano, Ed. Vallardi Milano 1911.

Nell'immagine sono indicati i tracciati originali del Seveso e dell'Olona e le loro probabili deviazioni (rispettivamente i tratti S'-T-T'-U' e C-D) intervenute in epoca romana per immetterli nel fossato di difesa che aveva come principale emissario a roggia Vettabbia.

Nel 1135 i frati Cistercensi, venuti a Milano al seguito di Bernardo di Chiaravalle, iniziarono la fondazione della loro Abbazia, fuori Porta Romana, sui terreni donati loro dai milanesi, incolti e acquitrinosi, dove si impaludavano le acque putride della Vettabbia.

Secondo una tradizione consolidata, i monaci delle due Abbazie di Chiaravalle e Viboldone, sorte in fregio alla Vettabbia, avviarono un'importante opera di bonifica del territorio circostante, incanalando e regolando lo scorrimento delle acque e sviluppando l'utilizzo dei prati marcitori. 
La tecnica, ancora in uso, consiste nel fare scorrere sul terreno un velo d'acqua in costante movimento, utilizzando generalmente l'acqua di risorgiva caratterizzata da una temperatura costante che nel periodo invernale protegge i campi dal gelo.

Ciò consente di estendere la produzione dei prati all'intero arco dell'anno e di poter disporre di foraggio fresco anche durante l'inverno.

In realtà è stato dimostrato come l'uso delle marcite risulti antecedente all'arrivo dei monaci che ne adottarono la pratica e sicuramente contribuirono a perfezionarla e a diffonderla su un ampio territorio a valle della città, utilizzando in particolare anche le acque luride della Vettabbia.

Queste acque, ricche di sostanze fertilizzanti (azoto, fosforo, potassio e calcio), scorrendo sul prato, subiscono un'intensa aerazione che favorisce la separazione delle sostanze nutrienti che si depositano sul terreno.

Le acque di colatura in uscita dal primo campo vengono raccolte e indirizzate al successivo appezzamento e così via.

Alla fine del loro percorso, attraversando in successione una serie dei prati marcitori, le acque di rifiuto subiscono una depurazione biologica naturale dopo aver fornito al terreno agricolo un benefico apporto di sostanze fertilizzanti (Fig. 2).

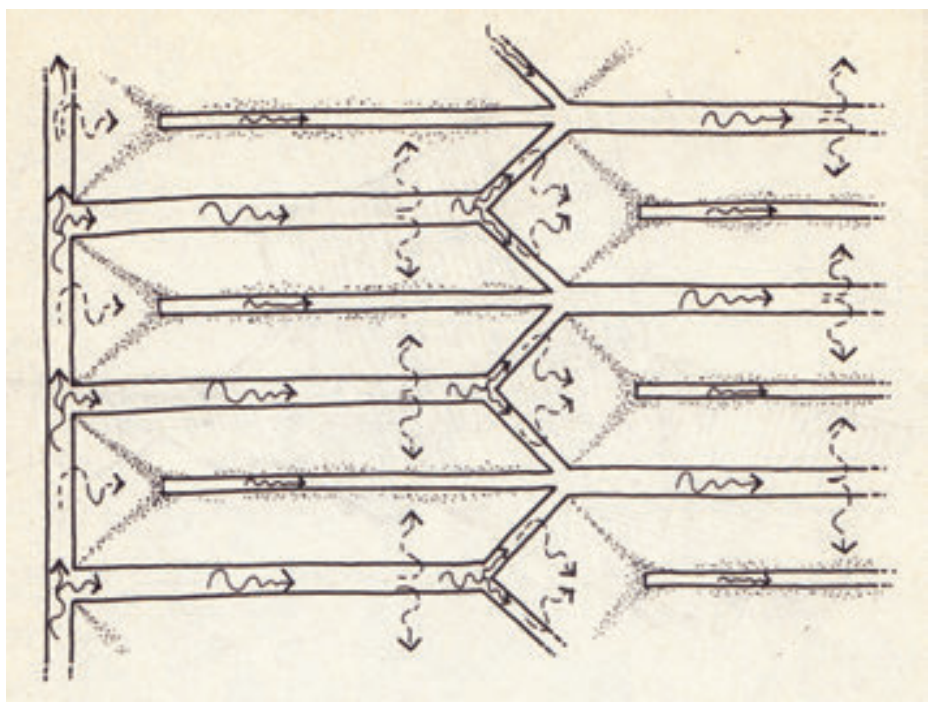

Fig. 2. Schema di marcita ad ali. 
In uno studio pubblicato nel 1932 il Prof. Claudio Antoniani del Laboratorio di Chimica Agraria del Regio Istituto Superiore Agrario di Milano evidenziava come la produzione media di foraggio di una marcita irrigata con acqua di fontanile fosse di $6-7$ tagli annuali rispetto ai $4-5$ di un prato stabile e che salisse anche fino a 9 -10 tagli per le marcite irrigate con acque cloacali.

\section{La fognatura di Milano e lo smaltimento Delle aCQue REFLUE URBANE}

Nella seconda metà del XIX secolo, sotto la spinta dell'espansione urbana, si affermò l'esigenza di realizzare un efficiente e moderno sistema fognario, a salvaguardia dell'igiene e della salute publica.

Dopo un ventennio di approfonditi studi che misero a confronto le esperienze e i sistemi adottati nelle principali città europee, nel 1890 fu approvato il "Progetto generale della rete di fognatura della città" redatto sotto la guida dell'Ing. Felice Poggi dell'Ufficio Tecnico Municipale.

Il Progetto prevedeva la realizzazione di un sistema di canalizzazioni indipendente dal preesistente reticolo dei corsi d'acqua, di tipo unitario, ovvero predisposto per raccogliere in unico condotto le acque di rifiuto e quelle di pioggia e funzionante per gravità, sfruttando la pendenza naturale del suolo.

Per lo smaltimento del consistente volume delle acque reflue provenienti dall'area urbana in rapida espansione, venne confermato l'utilizzo dei prati marcitori esistenti a valle della città, irrigati dalla Roggia Vettabbia.

La scelta venne avvallata, oltre che dagli indirizzi scientifici internazionali dell'epoca (Congresso d'Igiene di Parigi - 1889), anche da approfonditi studi di due Commissioni Municipali del 1890 e del 1901.

In particolare nell'ambito della Commissione del 1901, i professori Angelo Menozzi e Angelo Celli dimostrarono la validità del metodo di smaltimento dei reflui fognari sui prati marcitori, sia sotto l'aspetto dell'efficienza depurativa che di quello della sicurezza igienico sanitaria.

Venne stabilita anche la superficie ottimale del terreno destinato allo smaltimento dei reflui, pari a $90 \div 140$ abitanti per ettaro coltivato a marcita.

Si rese peraltro necessario incrementare la superficie di spandi- 
mento dei liquami per renderla compatibile con gli abitanti della città in rapido aumento.

A tale scopo fu stipulato un accordo con gli agricoltori riuniti nel Consorzio Utenti di Roggia Vettabbia, mediante due successive Convenzioni del 1889 e 1905.

In tal modo il Comune di Milano raggiunse lo scopo di smaltire le acque reflue della città, mentre gli utenti del Consorzio poterono usufruire di un benefico apporto di elementi fertilizzanti e della disponibilità sicura di acqua che incrementavano la loro produzione agricola.

Il sistema adottato fu oggetto di un'assidua vigilanza da parte dell'Ufficio d'Igiene di Milano, nonché di studi specialistici tra i quali ricordiamo quello già citato, prodotto nel 1932 dal Prof. Claudio Antoniani, che ne confermò la validità, sotto l'aspetto dell'apporto di elementi fertilizzanti, della produzione e qualità del foraggio, dell'efficienza depurativa e della sicurezza igienica.

Queste e successive valutazioni positive comportarono il mantenimento di questa pratica depurativa fino ai primi decenni del secondo dopoguerra.

Nel frattempo il sistema cominciava a perdere la sua efficacia a causa dell'espansione urbana e industriale, del conseguente incremento della popolazione, della contestuale riduzione della superficie dei terreni su cui attuare lo spandimento e della sempre più rilevante presenza di sostanze inquinanti provenienti dagli insediamenti produttivi.

\section{L'ATTUALE SISTEMA DI DEPURAZIONE DELLE ACQUE REFLUE DI MILANO}

La prima proposta di ricorrere a impianti di depurazione artificiali fu avanzata nel 1960 dall'Ing. Antonio Columbo, Vice Ingegnere Capo dell'Ufficio Tecnico Comunale.

La proposta prevedeva la realizzazione di due impianti di trattamento parziale, delle acque ordinarie, destinate comunque a una successiva depurazione biologica naturale, da collocare a monte dei due grandi recapiti storici delle acque di fognatura: la roggia Vettabbia e il colatore Lambro Meridionale.

Purtroppo ci vollero le prescrizioni dell'Unione Europea all'inizio degli anni ' 80 per disporre di un moderno ed efficiente sistema depurativo, realizzato sotto la guida di un Commissario governativo. 
Dal 2004 le acque reflue della città di Milano sono completamente trattate in tre impianti di depurazione, situati a valla della città, che servono i tre bacini scolanti secondo cui è suddiviso il territorio urbano: Milano San Rocco, Milano Nosedo e la seconda linea del depuratore di Peschiera Borromeo (Fig. 3).

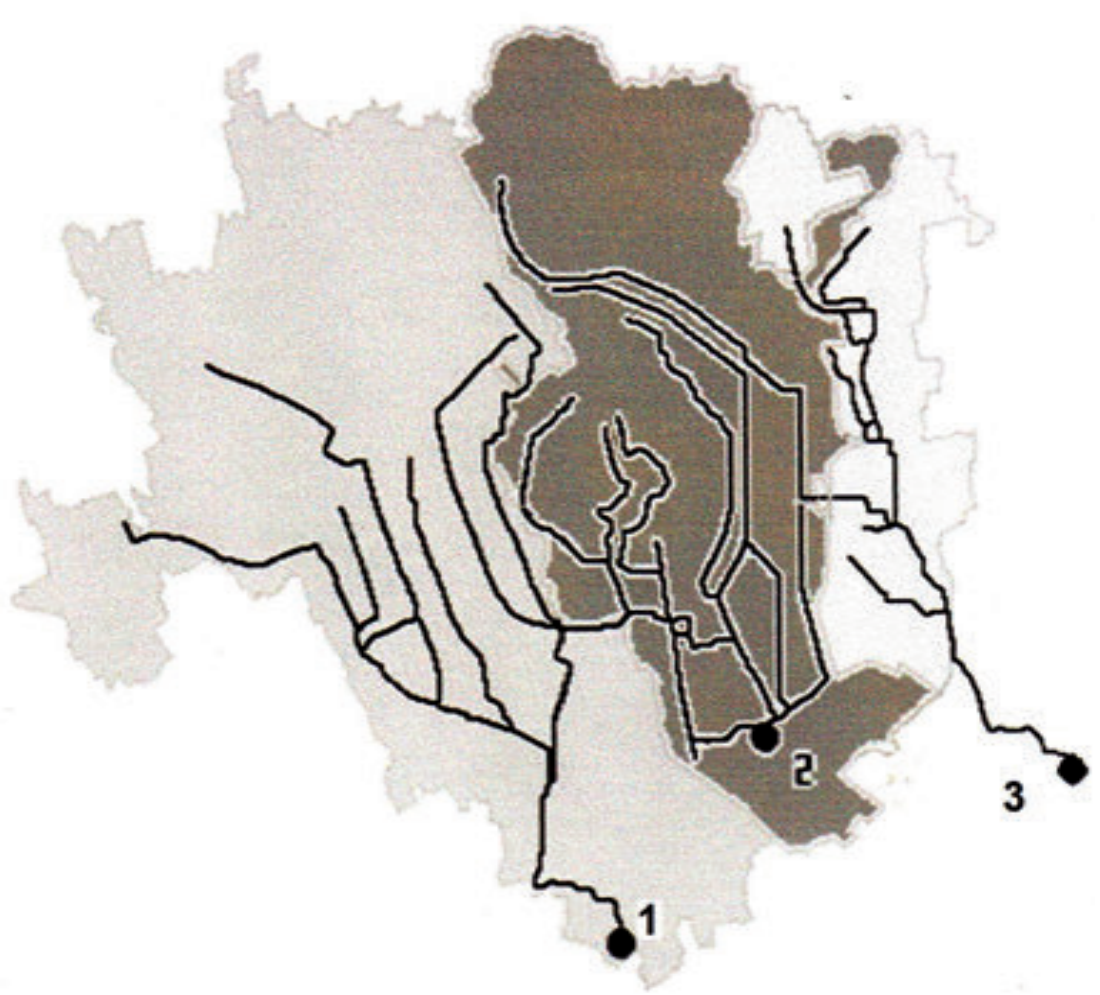

Fig. 3. Gli impianti di depurazione del Sistema di fognatura Milanese e i relativi bacini scolanti.

Il primo impianto, ubicato nelle vicinanze del borgo di Ronchetto delle Rane, in prossimità del confine tra Milano, Rozzano e Opera, tratta le acque reflue provenienti dal bacino Orientale con una superficie di circa 10.130 ettari, e di una parte di quelle provenienti dal territorio di Settimo Milanese. 
Ha una potenzialità pari a 1.050 .000 abitanti equivalenti e può trattare una portata di 4 metri cubi al secondo, in tempo asciutto, che può essere incrementata fino a 12 in periodo di pioggia.

L'impianto è stato progettato e realizzato dalla Società Dégremont S.p.A. che lo ha condotto fino al dicembre 2014, quando gli è subentrata MM Spa, a cui il Comune di Milano ha affidato nel 2003 la gestione del Servizio Idrico Integrato (acquedotto, fognatura e depurazione) della città.

Il secondo, Nosedo, che sorge nei pressi dell'omonimo abitato, a monte dell'Abbazia di Chiaravalle, opera a servizio del bacino Centroorientale, che è il più densamente abitato e ha un'estensione di circa 6.900 ettari.

Ha una potenzialità pari a 1.250 .000 abitanti equivalenti e può trattare una portata di 5 metri cubi al secondo, in tempo asciutto, che può essere incrementata fino a 15 in periodo di pioggia.

Il depuratore di Nosedo realizzato nell'ambito di una concessione di costruzione e gestione stipulata dal Comune di Milano con la società MilanoDepur S.p.A. è stato il primo grande depuratore della città a entrare in servizio.

Le acque raccolte nel bacino Orientale, con superficie pari a circa 2.230 ettari, sono invece recapitate all'impianto di Peschiera Borromeo, dove è stata realizzata una linea dedicata, con potenzialità di 250.000 abitanti equivalenti, in grado di trattare una portata di circa 1 metro cubo al secondo, in tempo asciutto, e di 3 metri cubi al secondo in periodo di pioggia.

Le caratteristiche generali del sistema sono riassunte in Tab. 1.

Tab. 1. Sommario delle caratteristiche degli impianti del sistema di depurazione delle acque reflue del Comune di Milano.

\begin{tabular}{lccc}
\hline Impianto & San Rocco & Nosedo & Peschiera B. \\
\hline Bacino & Occidentale & Centro-Orientale & Orientale \\
\hline Superficie [ha] & 10.130 & 6.900 & 2.230 \\
\hline Potenzialità [Abitanti Equiv.] & 1.050 .000 & 1.250 .000 & 250.000 \\
\hline Portata tempo asciutto $\left[\mathrm{m}^{3} / \mathrm{s}\right]$ & 4,00 & 5,00 & 1,10 \\
\hline Portata massima $\left[\mathrm{m}^{3} / \mathrm{s}\right]$ & 12,00 & 15,00 & 3,30 \\
\hline
\end{tabular}


Uno degli aspetti più interessanti del complesso depurativo di Milano è rappresentato dai limiti di emissione adottati per le acque depurate che sono tra i più restrittivi a livello sia italiano che europeo: non solo sono applicati i parametri previsti per le cosiddette "aree sensibili", ma addirittura quelli imposti dal Decreto del Ministero dell'Ambiente n. 185 del 2003, necessari per consentire il riuso in agricoltura delle acque depurate in agricoltura (Tab. 2).

Tab. 2. Limiti di emissione delle acque reflue trattate dagli impianti di depurazione di Milano.

\begin{tabular}{|c|c|}
\hline Parametri & Limiti di emissione \\
\hline$\overline{\mathrm{BOD}_{5}}$ & $\leq 10 \mathrm{mg} \mathrm{O} / \mathrm{l}^{\mathrm{a}}$ \\
\hline $\mathrm{COD}$ & $\leq 100 \mathrm{mg} \mathrm{O} / 1^{b}$ \\
\hline Solidi sospesi totali & $\leq 10 \mathrm{mg} / \mathrm{l}$ \\
\hline Azoto totale & $\leq 10 \mathrm{mg} \mathrm{N} / 1$ \\
\hline Azoto ammoniacale & $\leq 2 \mathrm{mg} \mathrm{NH}_{4} / \mathrm{l}$ \\
\hline Fosforo totale & $\leq 1 \mathrm{mg} \mathrm{P} / 1$ \\
\hline Escherichia coli & $\leq 10 \mathrm{UFC} / 100 \mathrm{mlc}^{\mathrm{c}}$ \\
\hline
\end{tabular}

Gli impianti che a tutti gli effetti attuano questa funzione sono i due principali, Nosedo e San Rocco, che trattano complessivamente più del 90\% delle acque reflue della città, mentre quelle residuali del bacino Orientale, depurate a Peschiera Borromeo, vengono attualmente recapitate nel fiume Lambro Settentrionale.

I processi depurativi dei due impianti di Nosedo e San Rocco sono sostanzialmente analoghi, del tipo biologico a fanghi attivi e il trattamento dei liquami è suddiviso in tre stadi.

Si inizia con lo stadio primario, dove il liquame grezzo raccolto dalla fognatura viene sottoposto a una serie di trattamenti meccanici e fisici che hanno la funzione di separare le sostanze solide grossolane (rifiuti galleggianti, stracci, residui alimentari, ecc.) le sabbie, gli oli e i grassi. 
Il processo è articolato secondo due fasi di grigliatura grossolana e fine, una fase di dissabbiatura e una di disoleazione.

Poi le acque vengono trasferite al secondo stadio, ovvero al trattamento biologico che ha lo scopo di separare le sostanze disciolte e sospese non sedimentabili.

Il liquame è immesso in grandi vasche, aerate mediante insufflazione d'aria dal fondo, per favorire il processo di demolizione della sostanza organica biodegradabile operato dalla biomassa batterica presente in sospensione (fanghi attivi).

La flora batterica si addensa in fiocchi, composti di batteri e materia organica, che tendono a sedimentare e quindi possono essere separati con facilità dall'acqua nelle successive vasche di sedimentazione finale.

Infine, le acque subiscono un ulteriore trattamento, definito terzo stadio, articolato nelle due fasi di filtrazione e disinfezione finalizzato all'eliminazione delle residue tracce di sostanze sospese, di azoto e fosforo, alla rimozione di detergenti sintetici e alla distruzione di microrganismi patogeni.

All'uscita degli impianti di depurazione le acque raggiungono un livello qualitativo elevato, tale da rispettare ampiamente i parametri di legge previsti per le aree sensibili e per l'uso in agricoltura.

I reflui trattati dall'impianto di Nosedo sono resi idonei al riutilizzo irriguo per l'intero arco dell'anno e costituiscono l'unica fonte di alimentazione del ramo inferiore della roggia Vettabbia che svolge la funzione irrigua su un vasto comprensorio agricolo tra Milano e Melegnano della superficie di circa 3.700 ettari.

Le acque depurate sono distribuite dal Consorzio Utenti di Roggia Vettabbia a 90 aziende agricole dove sono utilizzate per coltivare mais $(45 \%)$, riso $(15 \%)$, altri cereali e foraggi $(40 \%)$.

La potenzialità di distribuzione all'uso irriguo in tempo asciutto è attualmente di circa 3,4 metri cubi al secondo.

Le portate eccedenti sono recapitate nel cavo Redefossi, ma in un prossimo futuro anche queste acque saranno destinate agli usi agricoli grazie in particolare alla realizzazione a valle del depuratore del nuovo Parco agricolo urbano della Vettabbia: un territorio di circa 100 ettari.

In quest'ambito verrà anche ripristinata una marcita di fronte all'abbazia Chiaravalle e riattivato il canale che consentirà di muovere la ruota idraulica dell'antico mulino dell'Abbazia recentemente restaurato. 
Mediamente la portata annua di Nosedo effettivamente utilizzata a scopi irrigui è di circa 78,8 milioni di metri cubi.

Anche l'intero volume delle acque trattate in tempo asciutto dall'impianto di Milano San Rocco può essere resa compatibile per il riutilizzo agricolo, ma l'effettiva erogazione avviene solo durante la stagione irrigua.

In questo periodo l'intera portata viene consegnata al Consorzio di Bonifica Est Ticino Villoresi che provvede all'erogazione alle due rogge Carlesca e Pizzabrasa gestite da consorzi di utenti privati.

Le acque dell'impianto integrano sensibilmente la dotazione idrica delle due rogge, che irrigano una superficie agricola complessiva di circa 7.700 ettari che si estende nel sud-ovest milanese fino a interessare la provincia di Pavia.

Mediamente la portata annua di San Rocco utilizzata a scopi irrigui è di circa 11,4 milioni di metri cubi.

Nei mesi invernali le acque trattate sono scaricate nel colatore Lambro Meridionale che raccoglie anche le acque in esubero in tempo di pioggia.

In conclusione l'entrata in funzione dei depuratori di Milano ha avuto una positiva ricaduta su un ampio territorio a valle della città.

Innanzitutto la possibilità del riutilizzo a scopi irrigui delle acque di fognatura opportunamente depurate di Milano, ripristinando una pratica secolare, costituisce un importante fattore di sviluppo delle pratiche agricole periurbane (prodotti di qualità a chilometro zero).

Inoltre l'immissione di acque di buona qualità sull'ampio comprensorio agricolo di valle ha contribuito in maniera determinante al suo recupero ambientale e paesaggistico, nonché alla valorizzazione del patrimonio storico-culturale esistente (Abbazie di Chiaravalle e Viboldone, Borgo di Chiaravalle, Cascine storiche, Parco agricolo del Sud Milano).

Pertanto, il caso del Servizio Idrico Integrato della città di Milano dimostra come le acque reflue opportunamente depurate possono rappresentare una risorsa preziosa per l'agricoltura.

Esse costituiscono, infatti, una fonte sicura, per qualità e quantità costante tutto l'anno, utile a integrare la disponibilità dei corsi d'acqua naturali, che assume una valenza strategica nei periodi irrigui estivi e anche economicamente sostenibile.

Il costo dell'acqua a Milano, comprensivo di tutte le fasi del Servizio Idrico Integrato (captazione, trattamento e distribuzione del- 
l'acqua potabile, raccolta, collettamento e depurazione delle acque reflue, fino alla loro restituzione all'ambiente) è uno dei più bassi in Italia e in Europa.

\section{BIBLIOGRAFIA}

Menozzi Celli. Commissione per la Fognatura 1901 - All. IV - Stabilimento Tipografico Enrico Reggiani, Milano 1902.

Felice Poggi. La Fognatura di Milano. Antonio Vallardi, Milano 1911.

C. Antoniani, E. Sudario, L. Vianello. La depurazione agricola delle acque cloacali di Milano - Laboratorio di Chimica Agraria del R. Istituto Superiore Agrario, Milano 1932.

A. Gentile, M. Brown, G. Spadoni. Viaggio nel sottosuolo di Milano tra acque e canali segreti - Comune di Milano 1990.

IEFE. Il sistema di depurazione di Milano: dall'emergenza alla sostenibilità - Forum Editrice 2006. 